J. Austral. Math. Soc. (Series A) 29 (1980), 129-142

\title{
LIE AND JORDAN STRUCTURE IN OPERATOR ALGEBRAS
}

\author{
DEREK W. ROBINSON AND ERLING ST
}

(Received 31 July 1979)

Communicated by J. B. Miller

\begin{abstract}
Let $\mathfrak{U}$ be a $C^{*}$-algebra, $\alpha a^{*}$-anti-automorphism of order 2 , and $\mathfrak{A}^{\alpha}( \pm 1)=\{A ; A \in \mathfrak{A}, \alpha(A)=$ $\pm A\}$ the spectral subspaces of $\alpha$. It follows that $\mathfrak{A}^{\alpha}(+1)$ is a Jordan algebra and $\mathfrak{U}^{\alpha}(-1)$ is a Lie algebra. We begin the classification of pairs of Jordan and Lie algebras which can occur in this manner by examining $\mathfrak{A}=\mathscr{L}(\mathscr{H})$, the algebra of bounded operators on a Hilbert space $\mathscr{H}$.
\end{abstract}

1980 Mathematics subject classification (Amer. Math. Soc.): 46 L 99.

\section{Introduction}

Recently Effros and the second-named author (Effros and Størmer (1979)) began the analysis of Jordan structure associated with positive identity-preserving projections $P$ acting on a $C^{*}$-algebra $\mathfrak{A}$ with identity 1 . Thus $P ; A \in \mathfrak{U} \rightarrow P(A) \in \mathfrak{U}$ satisfies $P \geqslant 0, P^{2}=P$, and $P(1)=1$. The starting point of the analysis, and a subsequent paper (Størmer (1979)), is a proof that the range $P\left(\mathscr{A}_{h}\right)$ of $P$ on the hermitian elements $\mathfrak{U}_{h}$ of $\mathfrak{U}$ is a Jordan algebra when equipped with a suitable new product. One consequence of this result is that if $\mathfrak{A}$ is a von Neumann algebra and $\alpha$ a normal positive identity preserving map of $\mathfrak{X}$ into itself then the hermitian fixed points of $\alpha$ have a natural Jordan structure. An easy example of this latter result occurs if $\alpha$ is a *-automorphism, or a *-anti-automorphism, of $\mathfrak{A}$.

The starting point of the present note is the observation that if $\alpha$ is an antiautomorphism then the elements of $\mathfrak{A}$ satisfying $\alpha(A)=-A$ form a Lie-algebra with respect to the natural Lie product $[A, B]=A B-B A$. This follows from the

(C) Copyright Australian Mathematical Society 1980

Copyright. Apart from any fair dealing for scholarly purposes as permitted under the Copyright Act, no part of this JOURNAL may be reproduced by any process without written permission from the Treasurer of the Australian Mathematical Society. 
simple calculation

$$
\begin{aligned}
\alpha([A, B]) & =\alpha(A B)-\alpha(B A) \\
& =\alpha(B) \alpha(A)-\alpha(A) \alpha(B)=-[A, B] .
\end{aligned}
$$

Our purpose is to explore relations between this Lie structure, the Jordan structure of the fixed points, and positive projections associated with anti-automorphisms.

Suppose, more generally, that $\mathfrak{A}$ is an algebra over a field $k$ and $\alpha$ is a $k$-linear anti-automorphism of $\mathfrak{A}$ then the eigenspaces $\mathfrak{A}^{\alpha}( \pm 1)=\{A ; A \in \mathfrak{A}, \alpha(A)= \pm A\}$ are Jordan and Lie subalgebras of $\mathfrak{A}$ under the products

$$
A \circ B=(A B+B A) / 2,[A, B]=A B-B A,
$$

respectively. Moreover the linear span of $\mathfrak{U}^{\alpha}(+1)$ and $\mathfrak{U}^{\alpha}(-1)$ is a subalgebra $\mathscr{L}$ of $\mathfrak{A}$ on which $\alpha$ acts as an anti-automorphism of order 2 . This follows from the easily verified relations

$$
\begin{gathered}
{\left[\mathfrak{H}^{\alpha}(-1), \mathfrak{H}^{\alpha}(+1)\right] \subseteq \mathfrak{U}^{\alpha}(+1), \quad\left[\mathfrak{U}^{\alpha}(+1), \mathfrak{U}^{\alpha}(+1] \subseteq \mathfrak{U}^{\alpha}(-1),\right.} \\
\mathfrak{H}^{\alpha}(+1) \circ \mathfrak{U}^{\alpha}(-1) \subseteq \mathfrak{U}^{\alpha}(-1)
\end{gathered}
$$

which imply that $\mathscr{L}$ is both a Lie algebra and a Jordan algebra. Thus if one considers different powers of $\alpha$ and the corresponding eigenspaces one can reduce a substantial part of spectral theory for $\alpha$ to the study of anti-automorphisms of order 2, and the associated Jordan and Lie structure.

In this note we describe parts of the latter theory when $\mathfrak{A}$ is a $C^{*}$-algebra and $\alpha$ preserves the *-operation. Our principal result concerns the case that $\mathfrak{U}=\mathscr{L}(\mathscr{H})$. We show that there is a pairing between the classical Lie algebras and their infinite dimensional analogues, and the irreducible reversible Jordan algebras of self-adjoint operators or the scalars.

\section{Pairing of Lie and Jordan algebras}

We begin by examining some pairing properties of Lie and Jordan algebras in finite dimensions.

Let $M_{n}$ denote the $n \times n$ matrices and $\alpha$ a $*$-anti-automorphism of $M_{n}$. Since there is a unique normalized trace on $M_{n}$ it follows that

$$
\operatorname{Tr}(\alpha(A))=\operatorname{Tr}(A)
$$

for all $A \in M_{n}$. Consequently if $A \in M_{n}^{\alpha}(-1)$ and $B \in M_{n}^{\alpha}(+1)$

$$
\begin{aligned}
\operatorname{Tr}\left(A B^{*}\right) & =-\operatorname{Tr}\left(\alpha(A) \alpha\left(B^{*}\right)\right) \\
& =-\operatorname{Tr}\left(\alpha\left(B^{*} A\right)\right)=-\operatorname{Tr}\left(A B^{*}\right)
\end{aligned}
$$


and hence

$$
\operatorname{Tr}\left(A B^{*}\right)=0 \text {. }
$$

But $M_{n}$ is the linear span of $M_{n}^{\alpha}(+1)$ and $M_{n}^{\alpha}(-1)$, because each $A \in M_{n}$ has the decomposition

where

$$
A=A_{+}+A_{-}
$$

$$
A_{ \pm}=(A \pm \alpha(A)) / 2 \in M_{n}^{\alpha}( \pm 1) \text {. }
$$

Thus one deduces that the Jordan algebra $M_{n}^{\alpha}(+1)$ is the orthogonal complement $M_{n}^{\alpha}(-1)^{\perp}$ of the Lie algebra $M_{n}^{\alpha}(-1)$ when $M_{n}$ is equipped with the Hilbert space scalar product

$$
(A, B)=\operatorname{Tr}\left(A B^{*}\right)
$$

This motivates the examination of Lie subalgebras of $M_{n}$ which have Jordan subalgebras as their orthogonal complements, and vice versa.

The Cartan-Killing classification (Z-X Wan (1975)) of Lie subalgebras of $M_{n}$ is given in terms of four families of 'classical' algebras $A_{n}, B_{n}, C_{n}, D_{n}$, together with five 'exceptional' algebras $G_{2}, F_{4}, E_{6}, E_{7}, E_{8}$. These latter algebras will play no role in the sequel.

The algebra $A_{n-1}$ consists of the $n \times n$ matrices with trace zero. The algebras $B_{n}$ only occur if $n$ is odd and $C_{n}$ and $D_{n}$ occur if $n$ is even. The $B_{n}, C_{n}, D_{n}$ are identifiable as eigenspaces $M_{n}^{\alpha}(-1)$ of *-anti-automorphisms of the form

$$
A \rightarrow \alpha(A)=U A^{l} U^{-1}
$$

where $A^{l}$ denotes the transpose of $A$ and $U$ is a unitary matrix whose form varies with the class. The three possibilities $U_{B_{n}} U_{C_{n}}, U_{D_{n}}$, are usually expressed in the forms

$$
U_{B_{n}}=\left[\begin{array}{ccc}
1 & & 0 \\
& 0 & \mathbf{1}_{(n-1) / 2} \\
0 \mathbf{1}_{(n-1) / 2} & 0
\end{array}\right], \quad U_{C_{n}}=\left[\begin{array}{cc}
0 & \mathbf{1}_{n / 2} \\
-\mathbf{1}_{n / 2} & 0
\end{array}\right],
$$

$$
U_{D_{n}}=\left[\begin{array}{cc}
0 & \mathbf{1}_{n / 2} \\
\mathbf{1}_{n / 2} & 0
\end{array}\right]
$$

where $\mathbf{1}_{m}$ denotes the identity matrix in $M_{m}$. By repeated transposition of rows and columns one easily sees that these matrices are similar to the unitary matrices 


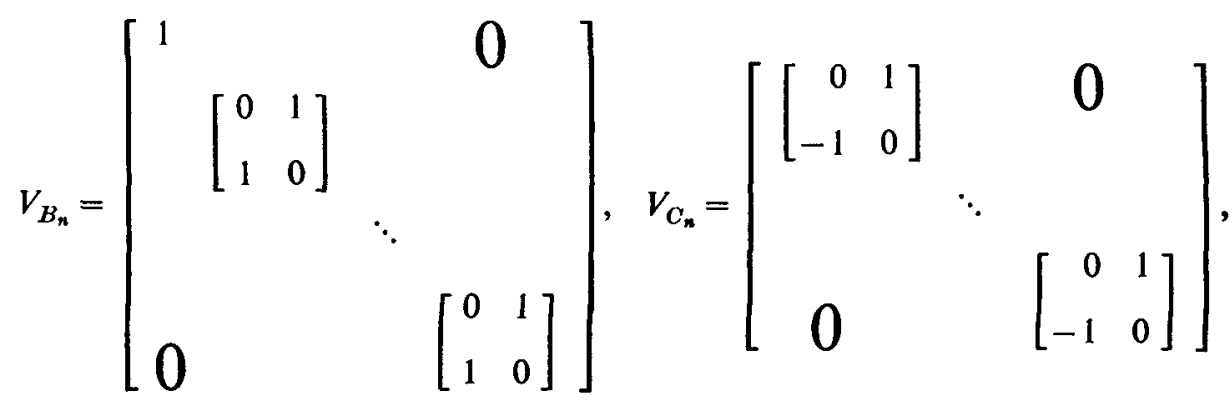

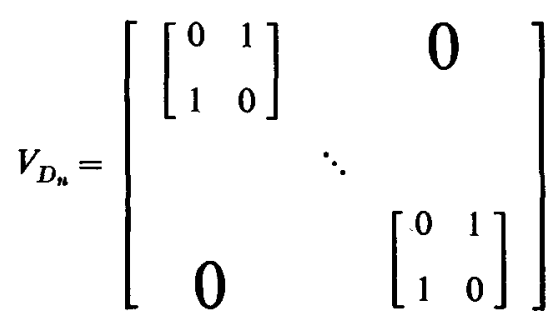

where the blocks $\left[\begin{array}{ll}0 & 1 \\ 1 & 0\end{array}\right]$ and $\left[\begin{array}{rr}0 & 1 \\ -1 & 0\end{array}\right]$ are in $M_{2}$. From this latter identification of $B_{n}, C_{n}, D_{n}$, it is readily calculated that the self-adjoint part of the orthogonal complements $B_{n}^{\perp}$, and $D_{n}^{\perp}$, are the real symmetric matrices $S_{n}$, whilst the selfadjoint part of $C_{n}^{\perp}$ is the Jordan algebra $Q_{n}$ of self-adjoint matrices whose entries are blocks of $2 \times 2$ matrices identified with the quaternions. Thus $B_{n}^{\perp}=S_{n}+i S_{n}$, $C_{n}^{\perp}=Q_{n}+i Q_{n}, D_{n}^{\perp}=S_{n}+i S_{n}$ and it is readily verified that $A_{n-1}^{\perp}=\mathbf{C l}$.

Now a self-adjoint Jordan algebra is defined to be simple if it has no proper nonzero self-adjoint Jordan ideals. Our first result establishes that the above classical algebras plus the trivial cases 0 and $M_{n}$ are the only Lie subalgebras of $M_{n}$ which have simple Jordan algebras as their orthogonal complements.

Proposition 1. Let $\mathfrak{g}$ be a self-adjoint Lie subalgebra of $M_{n}$ and assume that its orthogonal complement $\mathfrak{g}^{\perp}$ with respect to the Hilbert space scalar product

$$
(A, B)=\operatorname{Tr}\left(A B^{*}\right)
$$

is a Jordan algebra.

It follows that

1. $\left[\mathfrak{g}, \mathfrak{g}^{\perp}\right] \subseteq \mathfrak{g}^{\perp}$

2. $\mathfrak{g}^{\perp}$ is a simple Jordan algebra

3. $\mathfrak{g}$ is one of $0, A_{n-1}, B_{n}, C_{n}, D_{n}, M_{n}$.

Furthermore if $\mathrm{g} \circ \mathrm{g} \subseteq \mathrm{g}^{\perp}$ then $\mathrm{g}$ can only be one of $0, A_{1}, B_{n}, C_{n}, D_{n}$. 
Proof 1. Let $A, B \in \mathfrak{g}$ and $C \in \mathfrak{g}^{\perp}$ then

$$
\operatorname{Tr}([C, A] B)=\operatorname{Tr}(C[A, B])=0
$$

because $[A, B] \in \mathfrak{g}$. Thus $[C, A] \in \mathfrak{g}^{\perp}$ which establishes 1 .

2. Since $\mathrm{g}^{\perp}$ is a finite-dimensional self-adjoint Jordan algebra its simplicity will follow if we can establish that its only central projections are 0 and 1 .

Let $E$ be a central projection of $\mathfrak{g}^{\perp}$. Then if $A \in \mathfrak{g}$ we have

$$
E A E-A E=[E, A] E=E[E, A]=E A-E A E,
$$

where the second step uses part 1, that is $[E, A] \in \mathfrak{g}^{\perp}$ because $A \in \mathfrak{g}$ and $E \in \mathfrak{g}^{\perp}$. Thus

$$
E A+A E=2 E A E .
$$

Successive multiplication on the left and right by $E$ yields

$$
E A=E A E=A E
$$

and consequently $E \in \mathfrak{g}^{\prime}$. Thus

$$
E \in\left(\mathfrak{g}+\mathfrak{g}^{\perp}\right)^{\prime}=M_{n}^{\prime}=\mathbf{C 1} .
$$

3. It follows from the discussion preceding the proposition, and the observation that if $\mathrm{g}=0$, or $M_{n}$, then $\mathrm{g}^{\perp}=M_{n}$, or 0 , that the cases listed in part 3 are all possible. It remains to prove that this list is exhaustive.

Let us consider the various possibilities for the Jordan algebra $\mathfrak{g}^{\perp}$.

If $\mathrm{g}^{\perp}$ is irreducible and its self-adjoint part is $Q_{n}$ then $\mathfrak{g}\left(=\mathfrak{g}^{\perp \perp}\right)$ is $C_{n}$. But if $\mathfrak{g}^{\perp}$ is irreducible and its self-adjoint part is $S_{n}$ then $\mathfrak{g}$ is $B_{n}$ or $D_{n}$.

Next if $\mathfrak{g}^{\perp}$ is reducible there are two possibilities. Either the self-adjoint algebra $\mathfrak{A}$ generated by $\mathfrak{g}^{\perp}$ is simple or it is not.

In the first of these cases we can identify $M_{n}$ with $\mathfrak{A} \otimes M_{k}$, for some $k$, where $\mathfrak{g}^{\perp}$ is identified with $\mathrm{g}^{\perp} \otimes \mathrm{Cl}_{k}$. Now let $\left\{e_{i j} ; i, j=1,2, \ldots, k\right\}$ denote the usual matrix units in $M_{k}$, then it follows easily that the orthogonal complement of $\mathrm{g}^{\perp}$ within $\mathfrak{A}$ is a Lie algebra $h$ and

$$
\mathrm{g}=\left\{A ; A=\sum_{i, j=1}^{k} A_{i j} \otimes e_{i j}, \sum_{i=1}^{k} A_{i i} \in h\right\} .
$$

Thus if $A, B \in \mathfrak{A}$ then $A \otimes e_{12}$ and $B \otimes e_{21}$ belong to $\mathfrak{g}$ so that

$$
A B \otimes e_{11}-B A \otimes e_{22}=\left[A \otimes e_{12}, B \otimes e_{21}\right] \in \mathfrak{g} .
$$

Hence $[A, B] \in h$. Thus $h$ contains all the commutators in $\mathfrak{A}$ and $h$ must be 
$A_{m-1} \otimes M_{k}$ for some $m$. But then

$$
A=\sum_{i, j=1}^{k} A_{i j} \otimes e_{i j} \in \mathfrak{g}
$$

if, and only if,

$$
\operatorname{Tr}\left(\sum_{i=1}^{k} A_{i i}\right)=0
$$

that is $\operatorname{Tr}(A)=0$ and $\mathfrak{g}=A_{n-1}$.

Next assume $\mathfrak{U}$ is not simple. Then it has exactly two nontrivial central projections $E$ and $F=1-E$ (see, for example, Jordan and others (1934) or Størmer (1968)) and $\mathfrak{U} E=\mathfrak{g}^{\perp} E$. Now we argue that $E M_{n} E \cap \mathfrak{g}$ is the orthogonal complement of $E \mathfrak{g}^{\perp}$ in $E M_{n} E$. Indeed if $E A E \in \mathfrak{g}$ then

$$
\operatorname{Tr}((E A E) E B)=\operatorname{Tr}(E A E B)=0
$$

for all $B \in \mathrm{g}^{\perp}$. Thus $E A E \in\left(E \mathrm{~g}^{\perp}\right)^{\perp}$. Conversely if $E A E \in\left(E \mathfrak{g}^{\perp}\right)^{\perp}$ then for all $B \in \mathfrak{g}^{\perp}$ one has

$$
0=\operatorname{Tr}((E A E) E B)=\operatorname{Tr}(E A E B)
$$

so $E A E \in \mathrm{g}$.

Next remark that since $E \mathfrak{g}^{\perp}=E \mathfrak{A}$ is simple, and of the form $M_{m} \otimes \mathbf{C 1}_{k}$, the arguments in the earlier part of the proof establish that $\left(E \mathrm{~g}^{\perp}\right)^{\perp}=E M_{n} E \cap \mathrm{g}=0$ or $A_{k-1}$. In the latter case $\mathfrak{g}^{\perp}$ is the scalars and $\mathfrak{U}$ cannot have two central projections. Thus we are left with the possibility $E M_{n} E \cap \mathfrak{g}=0$ or, equivalently, $E \mathfrak{g}^{\perp}=E \mathfrak{U}=E M_{n} E$. Now $E \mathfrak{U} \simeq F \mathfrak{U}($ Størmer (1968)) and by symmetry

$$
F \mathfrak{g}^{\perp}=F=F M_{n} F .
$$

Thus $n=2 m$ is even and we can make the identifications $\mathfrak{U}=M_{m} \oplus M_{m}$ with $\mathfrak{g}^{\perp}=\left\{A \oplus A^{t} ; A \in M_{m}\right\}$. Writing elements of $\mathfrak{A}, \mathfrak{g}$, etcetera as $2 \times 2$ matrices over $M_{m}$ one readily computes that

$$
\mathfrak{g}=\left\{\left[\begin{array}{cc}
A & B \\
C & -A^{\mathfrak{l}}
\end{array}\right] ; \quad A, B, C \in M_{m}\right\} .
$$

But this is a Lie algebra if, and only if, $m=1$. Thus $\mathfrak{g}=A_{1}$ and $\mathfrak{g}^{\perp}=\mathbf{C 1}$.

It now follows from the structure theory of simple self-adjoint Jordan algebras (Jordan and others (1934); Størmer (1966)) that the only remaining possibility is that the self-adjoint part of $\mathfrak{g}^{\perp}$ is a spin factor of real dimension greater than 6 and $n=2^{k}$ for some $k \geqslant 3$ (see, for example, Effros and Størmer (1979) or Størmer (1966)). Now let $E$ be a nonzero minimal projection in $\mathfrak{g}^{\perp}$ and remark that if $A \in \mathrm{g}^{\perp}$ and $B \in \mathrm{g}$ then

$$
\operatorname{Tr}(A(E B E))=\operatorname{Tr}((E A E) B)=0
$$


Hence $E \mathfrak{g} E \subseteq\left(E \mathfrak{g}^{\perp} E\right)^{\perp}$. But conversely if $E A E \in\left(E \mathfrak{g}^{\perp} E\right)^{\perp}$ and $B \in \mathfrak{g}^{\perp}$ then

$$
\operatorname{Tr}((E A E) B)=\operatorname{Tr}((E A E) E B)=0
$$

and hence $\left(E \mathfrak{g}^{\perp} E\right)^{\perp} \cap E M_{n} E \subseteq E \mathfrak{g} E$. Thus $E \mathfrak{g} E$ is the orthogonal complement of $E \mathrm{~g}^{\perp} E$ in $E M_{n} E$. But since $E$ is minimal in $\mathrm{g}^{\perp}$ one has $E \mathfrak{g}^{\perp} E=\mathrm{C} E$ and consequently $E \mathrm{~g} E=(\mathrm{C} E)^{\perp} \cap E M_{n} E$. But $F=1-E$ is also a minimal projection in $\mathrm{g}^{\perp}$ and thus one concludes that $E \mathfrak{g} E \simeq F \mathrm{~g} F \simeq A_{n / 2-1}$. Now choose $A \in E \mathrm{~g} E$ to be rank one and let $S \in \mathfrak{g}^{\perp}$ be a self-adjoint unitary such that

$$
S E S=F .
$$

It follows from part 1 of the proposition that $[S, A] \in \mathfrak{g}^{\perp}$. But $[S, A]$ has rank less than or equal to two and the spin algebra $\mathfrak{g}^{\perp}$ cannot contain a nonzero matrix of rank less than four. This is a contradiction and consequently $\mathfrak{g}^{\perp}$ cannot be a spin factor of dimension greater than 6 .

The final statement of the proposition is immediate because the condition $A \circ B \in \mathfrak{g}^{\perp}$ for all $A, B \in \mathfrak{g}$ excludes the possibilities $A_{n-1}$ with $n \geqslant 3$ and $M_{n}$.

COROLlaRY 2. If $\mathfrak{g}$ is a self-adjoint exceptional Lie subalgebra of $M_{n}$ then $\mathfrak{g}^{\perp}$ cannot be a Jordan algebra.

This is a partial reiteration of statement 3 of Proposition 1.

COROLlary 3. If $\mathrm{g}$ is a nonzero self-adjoint Lie subalgebra of $M_{n}$ then there is a *-anti-automorphism $\alpha$ of order 2 of $M_{n}$ with $\mathfrak{g}=M_{n}^{\alpha}(-1)$ and $\mathfrak{g}^{\perp}=M_{n}^{\alpha}(+1)$ if, and only if, $\mathrm{g}^{\perp}$ is a Jordan algebra and

$$
\mathfrak{g} \circ \mathfrak{g} \subseteq \mathfrak{g}^{\perp}
$$

Proof. If $\mathrm{g}=M_{n}^{\alpha}(-1)$ for some $\alpha$ and $A, B \in \mathfrak{g}$ then

$$
\alpha(A \circ B)=(\alpha(A B)+\alpha(B A)) / 2=A \circ B .
$$

Thus $\mathfrak{g} \circ \mathrm{g} \subseteq M_{n}^{\alpha}(+1)=\mathfrak{g}^{\perp}$. Conversely if this latter condition is satisfied then Proposition 1 implies that $g$ is one of the algebras $A_{1}, B_{n}, C_{n}, D_{n}$. The existence of an $\alpha$ in the cases $B_{n}, C_{n}, D_{n}$, follows from the definitions of these algebras whilst the case $n=2$ and $\mathfrak{g}=A_{1}$, is dealt with by defining $\alpha$ such that

$$
\alpha\left(\left[\begin{array}{ll}
a & b \\
c & d
\end{array}\right]\right)=\left[\begin{array}{cc}
d & -b \\
-c & d
\end{array}\right] .
$$

\section{Projections on $\mathscr{L}(\mathscr{H})$}

Next we examine the pairing of infinite-dimensional Jordan and Lie algebras but with a slight change of emphasis. If $\alpha$ is a $*$-anti-automorphism of order 2 of 
a $C^{*}$-algebra $\mathfrak{U}$ with identity and $\iota$ is the identity map then $P=(\iota+\alpha) / 2$ is a positive projection which preserves the identity. The eigenspaces $\mathfrak{Q}^{\alpha}(+1)$, and $\mathfrak{A}^{\alpha}(-1)$, now correspond to the fixed points of $P$, and the null space of $P$, respectively. Thus we analyze the corresponding pairing problem for projections. This approach is related to the work of Arazy and Friedman (1978) who discussed the characterization of all contractions of the compact operators $\mathscr{L} \mathbf{C}(\mathscr{H})$ on a separable Hilbert space and it is also a natural extension of parts of Effros and Størmer (1979) and Størmer (1979). First it is necessary to introduce Lie algebras which are infinite-dimensional analogues of the $B_{n}, C_{n}, D_{n}$.

The weakly closed Jordan subalgebras of $\mathscr{L}(\mathscr{H})$ have been classified (Størmer (1966); Størmer (1972)) in terms of four JW-factors, real symmetric factors, quaternionian factors, spin factors and hermitian parts of von Neumann algebras. In the finite-dimensional discussion of the previous section the first two occurred in association with classical Lie algebras and we next introduce infinite-dimensional analogues of the Lie algebras by generalizing this association. One natural method of introducing these infinite-dimensional Lie algebras is by orthogonality with respect to the trace on $\mathscr{L}(\mathscr{H})$ but this leads to annoying details of approximation of trace-class operators. A second method is through spectral theory of $*$-anti-automorphisms. The real symmetric irreducible JW-factor $\mathfrak{A}_{r}$ is the hermitian (self-adjoint) elements of $\mathscr{L}(\mathscr{H})$ which are invariant under a *-anti-automorphism of order 2 , the transpose map $A \rightarrow A^{t}$. Thus $\mathfrak{A}_{r}=\mathscr{L}(\mathscr{H})^{l}(+1)_{h}$. The eigenspace $\mathfrak{A}_{r}=\mathscr{L}(\mathscr{H})^{l}(-1)$ is a Lie algebra $\mathfrak{g}$ with the bracket operation $[A, B]=A B-B A$. If $\mathscr{H}$ has finite dimension $n$ then $\mathfrak{g}=B_{n}$ for $n$ odd and $\mathfrak{g}=D_{n}$ for $n$ even. If $\mathscr{H}$ is infinite dimensional we denote the algebra $g$ by $B_{\infty}\left(\right.$ or $D_{\infty}$ ). Similarly, the quaternion algebra $\mathfrak{A}_{q}$ is formed by the elements of $\mathscr{L}(\mathscr{H})$ which are invariant under a *-anti-automorphism $A \rightarrow A^{q}$. If $\mathscr{H}$ has finite dimension $n$ the associated Lie algebra $\mathfrak{g}=\mathscr{L}(\mathscr{H})^{q}(-1)$ is $C_{n}$ and hence we denote the infinite-dimensional analogue by $C_{\infty}$.

The connection between $B_{\infty}, C_{\infty}$ and $B_{n}, C_{n}$ can be made more precise by considering an $n$-dimensional projector $E \in \mathfrak{A}_{r}$, or $E \in \mathfrak{U}_{q}$. It follows immediately from the calculations of the previous section that $E \mathfrak{g} E=(E \mathfrak{U} E)^{\perp}$ in $E \mathscr{L}(\mathscr{H}) E$ and $E \mathrm{~g} E$ is isomorphic to $B_{n}, C_{n}$ or $D_{n}$. The first case occurs if $n$ is odd and $E \in \mathfrak{A}_{r}$, the second if $E \in \mathfrak{A}_{q}$, and the third if $n$ is even and $E \in \mathfrak{A}_{r}$.

TheOREM 4. Let $\mathscr{H}$ be a complex Hilbert space and $P$ a positive normal identity preserving projection of $\mathscr{L}(\mathscr{H})$ into itself such that $\mathfrak{A}=P\left(\mathscr{L}(\mathscr{H})_{h}\right)$ is a JC-algebra. Let $\mathrm{g}$ denote the null space of $P$.

1. If $n=\operatorname{dim} \mathscr{H}<+\infty$ then $\mathrm{g}$ is a Lie algebra if and only if either $\mathfrak{U}=\mathbf{R 1}$, in which case $\mathfrak{g}=A_{n-1}$ or $\mathfrak{A}$ is reversible and irreducible, in which case $\mathfrak{g}$ is one of $0, B_{n}, C_{n}, D_{n}$. 
2. If $\mathscr{H}$ is infinite-dimensional then $\mathfrak{g}$ is a Lie algebra if and only if $\mathfrak{A}$ is reversible and irreducible, in which case $\mathrm{g}$ is one of $0, B_{\infty}, C_{\infty}$.

Proof. If $n=\operatorname{dim} \mathscr{H}<+\infty, \mathfrak{U}=\mathbf{R 1}$ and $\mathfrak{g}=A_{n-1}$ then $P$ is a state with null space $A_{n-1}$ and hence $P$ is the trace. Next assume $\mathfrak{A}$ is irreducible and reversible. One can have $\mathfrak{A}=\mathscr{L}(\mathscr{H})_{h}, P=\imath$ and $\mathfrak{g}=0$. Alternatively $\mathfrak{A}$ could be the real symmetric operators or the quaternionian algebra. In both cases $P$ is unique by Størmer (1979), Lemma 6.1 and hence $P=(\imath+\alpha) / 2$ where $\alpha$ is the ${ }^{*}$-antiautomorphism of $\mathscr{L}(\mathscr{H})$ such that $\alpha(A)=A$ for $A \in \mathfrak{A}$. Consequently

$$
\mathfrak{g}=\mathscr{L}(\mathscr{H})^{\alpha}(-1)=\mathfrak{A}^{\perp}
$$

is $B_{n}, C_{n}$ or $D_{n}$, by Proposition 1 , or if $\operatorname{dim} \mathscr{H}=\infty$ by the definition of $B_{\infty}, C_{\infty}$. Conversely assume $g$ is a Lie algebra.

We first argue that $P$ must be faithful. Let $r=\operatorname{supp} P$ and assume $r \neq 1$. Let $A=r A(1-r)$ be nonzero in $\mathscr{L}(\mathscr{H})$. Then $P(A)=0$ and $A \in \mathrm{g}$. Since $P$ is selfadjoint $A^{*} \in \mathfrak{g}$ thus $r A(1-r) A^{*} r-(1-r) A^{*} r A(1-r)=\left[A, A^{*}\right] \in \mathfrak{g}$. Now

$$
(1-r) A^{*} r A(1-r) \in \mathfrak{g}
$$

and consequently $r A(1-r) A^{*} r \in g$. But $P$ is faithful on $r \mathscr{L}(\mathscr{H}) r$ and hence $r A(1-r) A^{*} r=0$ and $r A(1-r)=0$ contrary to assumption. Thus $r=1$ and $P$ is faithful.

Since $P$ is ultra-weakly continuous $\mathfrak{A}$ is a $\mathrm{JW}$-algebra. Let $l$ denote its centre, then $l+i l$ is an abelian von Neumann algebra and there is a complete family of normal projections of $\mathfrak{A}^{\prime \prime}$ onto $l+i l$ and in particular of $\mathfrak{U}+i \mathfrak{U}$ onto $l+i l$ (see the proof of $3 \Rightarrow 5$ in Størmer (1972)). Composing these projections with the faithful projection $P$ one obtains a complete family of normal projections of $\mathscr{L}(\mathscr{H})$ onto $l+i l$. Thus by Størmer (1972) the centre $l$ is totally atomic.

Next we argue that $\mathfrak{A}$ is a JW-factor. If not there exist two non-zero minimal projections $E \neq F$ in $l$. Let $[\mathfrak{U}]=P(\mathscr{L}(\mathscr{H}))$ denote the complexification $\mathfrak{A}+i \mathfrak{U}$ of $\mathfrak{A}$, then $\mathscr{L}(\mathscr{H})=[\mathfrak{U}]+\mathfrak{g}$, and each $B \in \mathscr{L}(\mathscr{H})$ has a decomposition $B=A+G$ with $A \in \mathfrak{U}$ and $G \in \mathfrak{g}$. But $E A F=0$ and $P(A \circ G)=A \circ P(G)$ by Størmer (1979), Lemma 4.1. Thus

$$
\begin{aligned}
P(E G F) & =P(E(E G F)+(E G F) E) \\
& =2 P(E \circ(E G F)) \\
& =2 E \circ P(E G F) .
\end{aligned}
$$

By symmetry

$$
P(E G F)=2 F_{\circ} P(E G F)
$$

and consequently

$$
P(E G F)=0 \text {. }
$$


Thus $E \mathscr{L}(\mathscr{H}) F \subset \mathfrak{g}$. Now assuming $\operatorname{dim} E \leqslant \operatorname{dim} F$ one can find a partial isometry $V \in E \mathscr{L}(\mathscr{H}) F$ such that $V V^{*}=E$ and $V^{*} V \leqslant F$. Since $V \in \mathfrak{g}, V^{*} \in \mathfrak{g}$ and $\left[V, V^{*}\right] \in \mathfrak{g}$. Now by Størmer (1979), Lemma 4.1, $P(E G E)=E P(G) E$ for $G \in \mathfrak{g}$, thus $E \mathfrak{g} E \subseteq \mathfrak{g}$ and

$$
E=V V^{*}=E\left[V, V^{*}\right] E \in \mathfrak{g}
$$

which is a contradiction. Thus $\mathfrak{A}$ is a JW-factor.

Next we claim that $\mathfrak{X}^{\prime}$ is a finite von Neumann algebra and $\left.P\right|_{\mathscr{H}^{\prime}}$ is a trace. Indeed if $B \in \mathfrak{A}^{\prime}$ then $B=A+G$ with $A \in[\mathfrak{U}]$ and $G \in \mathfrak{g}$. But $P(B)=\lambda \mathbf{1}$ and $\lambda \in \mathbf{C}$ by Størmer (1979), Lemma 4.2. Thus $A=P(B)=\lambda 1$ and $B=G+\lambda 1$. Now let $B^{\prime}=G^{\prime}+\lambda^{\prime} \mathbf{1}$ be another operator in $\mathfrak{Y}^{\prime}$ then

and

$$
\left[B, B^{\prime}\right]=\left[G, G^{\prime}\right] \in \mathrm{g}
$$

$$
P\left(\left[B, B^{\prime}\right]\right)=0 .
$$

So $P$ is a faithful normal trace on $\mathfrak{A}^{\prime}$. But if $B^{\prime}=G^{\prime}+\lambda^{\prime} \mathbf{1} \in \mathfrak{A}^{\prime}$ and $A \in[\mathfrak{U}], G \in \mathfrak{g}$ then

$$
\begin{aligned}
P\left(B^{\prime}(A+G)\right) & =P\left(A B^{\prime}+\left(G^{\prime}+\lambda^{\prime} \mathbf{1}\right) G\right) \\
& =P\left(A B^{\prime}+\left[G^{\prime}, G\right]+G G^{\prime}+\lambda^{\prime} G\right) \\
& =P\left((A+G) B^{\prime}\right) .
\end{aligned}
$$

Consequently if $\omega$ is any normal state on $\mathfrak{U}$ then $\omega \circ P$ is a normal state containing $\mathfrak{U}^{\prime}$ in its centralizer and the support of $\omega \circ P$ belongs to $\left(\mathfrak{U}^{\prime}\right)=\mathfrak{U}^{\prime \prime}$. Let $F=\operatorname{supp} \omega \circ P$ and let $\mathscr{H}$ be the centralizer of $\omega_{\circ} P$ in $\mathscr{L}(\mathscr{H})$. By Tomita's modular theory there exists a faithful normal projection $P_{\omega}$ of $F \mathscr{L}(\mathscr{H}) F$ onto $M F$. Since $M F$ is a finite von Neumann algebra containing $\mathfrak{U}^{\prime} F$ as a subalgebra there is a faithful normal projection $Q_{\omega}$ of $M F$ onto $\mathfrak{U}^{\prime} F$ and since $M F$ is of type I $\mathfrak{U}^{\prime} F$ is of type I (see Tomiyama (1959) or Størmer (1972)). Now $P$ is faithful and hence the states of the form $\omega \circ P$ form a complete set of normal states on $\mathscr{L}(\mathscr{H})$ and their supports are separating for $\mathfrak{U}^{\prime}$ so that $\mathfrak{U}^{\prime}$ is of type $\mathbb{I}$. But then $\mathfrak{I}^{\prime \prime}$ is of type $I$ and therefore $\mathfrak{U}$ is of type I (Størmer (1968)).

Our next aim is to prove that $\mathfrak{A}$ is reversible. Assume the contrary, then $\mathfrak{A}$ is a spin factor. Let $E$ and $F=1-E$ be minimal projections in $\mathfrak{A}$, then $E \mathfrak{g} E$ is a Lie subalgebra of $g$ and $P$ restricted to $E \mathscr{L}(\mathscr{H}) E$ is a state $\omega$, that is

$$
P(E A E)=\omega(E A E) E \text {. }
$$

But a state $\omega$ on a $C^{*}$-algebra $\mathscr{B}$ has a Lie algebra $\mathscr{L}$ as null space if and only if $\omega$ is a trace. Indeed if $A, B \in \mathscr{B}$ then $A-\omega(A) 1, B-\omega(B) 1 \in \mathscr{L}$. But then 


$$
[A, B]=[A-\omega(A) 1, B-\omega(B) 1] \in \mathscr{L}
$$

and hence

$$
\omega([A, B])=0
$$

for all $A, B \in \mathscr{L}$. In particular $\left.P\right|_{E \mathscr{L}(\mathscr{H}) E}$ is a trace so $\operatorname{dim} \mathscr{H}<+\infty$. Now $P(E A F)=0$ for all $A \in \mathscr{L}(\mathscr{H})$ by the earlier computation which showed that

$$
P(E A F)=2 E \circ P(E A F)=2 F_{\circ} P(E A F)
$$

But since $P$ is a trace on both $E \mathscr{L}(\mathscr{H}) E$ and $F \mathscr{L}(\mathscr{H}) F$ one must have

$$
\operatorname{Tr}(A)=\operatorname{Tr}(P(A))
$$

for all $A \in \mathscr{L}(\mathscr{H})$. In particular if $G \in \mathfrak{g}$ and $A \in[\mathfrak{U}]$ one has from Effros and Størmer (1979), Lemma 1.1 that

$$
\begin{aligned}
\operatorname{Tr}(G \circ A) & =\operatorname{Tr}(P(G \circ P(A))) \\
& =\operatorname{Tr}(P(G) \circ P(A))=0
\end{aligned}
$$

because $P(G)=0$. Thus $g \subseteq \mathfrak{U}^{\perp}$. But $\operatorname{dim} \mathscr{H}<+\infty$ and $\mathscr{L}(\mathscr{H})=\mathfrak{g}+[\mathfrak{A}]$ and hence $\mathfrak{g}=\mathfrak{A} \perp$. But by Proposition 1 this is impossible if $\mathfrak{U}$ is a nonreversible spin factor. Therefore $\mathfrak{U}$ is reversible.

There are now two cases to consider. Either $\mathfrak{U}^{\prime \prime}$ is a factor or $\mathfrak{U}^{\prime \prime}$ has exactly two nonzero central projections different from 1 (Størmer (1968)). Let us first assume $\mathfrak{A}^{\prime \prime}$ is a factor. Since $\mathfrak{U}^{\prime \prime}$ is of type $\mathrm{I}$ we can identify $\mathscr{L}(\mathscr{H})$ with $\mathfrak{U}^{\prime \prime} \otimes N$ where $N$ is isomorphic to $\mathfrak{U}^{\prime}$ and is a type $\mathrm{I}_{n}$ factor with $n<+\infty$. If $n=1$ then $N=\mathbf{C}$ and $\mathfrak{U}^{\prime \prime}=\mathscr{L}(\mathscr{H})$ and $P$ is the unique normal projection of $\mathfrak{U}^{\prime \prime}$ onto [थ] (see Størmer (1979), Proposition 6.2). Either $\mathfrak{g}=0$ if $[\mathfrak{U}]=\mathscr{L}(\mathscr{H}), \mathfrak{g}=B_{n}$ or $D_{n}$ if $n \in \mathbf{Z}_{+} \cup\{+\infty\}$ and $\mathfrak{U}$ is the real symmetric operators, and $\mathfrak{g}=C_{n}$ if $\mathfrak{U}$ is the quaternionian irreducible JW-factor. If $n \geqslant 2$ then Proposition 6.2 of Størmer (1979) establishes that $P=Q \otimes \tau$ where $Q$ is the unique normal projection of $\mathfrak{U}^{\prime \prime}$ onto [थ] and $\tau$ is the normalized trace on $\mathfrak{U}^{\prime}$. Let $\left\{e_{i j} ; i, j=1,2, \ldots, n\right\}$ be a complete set of matrix units in $N$, then if

$$
A=\sum_{i, j=1}^{n} A_{i j} \otimes e_{i j} \in \mathfrak{U}^{\prime \prime} \otimes N
$$

one has

$$
\begin{aligned}
P(A) & =P\left(\sum_{i, j=1}^{n} A_{i j} \otimes e_{i j}\right) \\
& =\frac{1}{n} Q\left(\sum_{i=1}^{n} A_{i i}\right) .
\end{aligned}
$$


Thus

$$
\mathfrak{g}=\left\{A ; A=\sum_{i, j=1}^{n} A_{i j} \otimes e_{i j}, \sum_{i=1}^{n} A_{i i} \in Q^{-1}(0)\right\}
$$

and in particular $A \otimes e_{12}, B \otimes e_{21} \in \mathfrak{g}$ for all $A, B \in \mathfrak{A}^{\prime \prime}$. Thus

and

$$
A B \otimes e_{11}-B A \otimes e_{22}=\left[A \otimes e_{12}, B \otimes e_{21}\right] \in \mathfrak{g}
$$

that is

$$
A B-B A=[A, B] \in Q^{-1}(0),
$$

$$
Q([A, B])=0 .
$$

Hence if $\omega$ is any normal state on $\mathfrak{A}, \omega \circ Q$ is a trace on $\mathfrak{A}^{\prime \prime}$ and consequently $\mathfrak{U}^{\prime \prime}$ is finite-dimensional. In praticular $Q$ itself must be the trace and $\mathfrak{g}=A_{m}$ for some $m$.

Finally assume $\mathfrak{U}^{\prime \prime}$ has exactly two minimal central projections $E$ and $F$ different from 1. Since $P$ is faithful $P(E)=\lambda 1$ with $0<\lambda<1$ (Størmer (1979), Lemma 4.2) and the map $P_{E} ; E \mathscr{L}(\mathscr{H}) E \rightarrow[\mathfrak{U}] E=\mathfrak{U}^{\prime \prime} E$ defined by

$$
P_{E}(E A E)=\frac{1}{\lambda} P(E A E) E
$$

is a faithful normal projection such that

$$
P_{E}(E)=E
$$

The null space $P_{E}^{-1}(0)$ is equal to $g \cap E \mathscr{L}(\mathscr{H}) E$ because $P_{E}(E A E)=0$ if and only if $P(E A E)=0$. Thus $P_{E}^{-1}(0)$ is a Lie algebra. Since $\mathfrak{A}^{\prime \prime} E$ is a type $I$ factor it is of the form $\mathscr{L}(\mathscr{K}) \otimes \mathbf{C 1}_{\mathscr{M}}$ where $\mathscr{K} \otimes \mathscr{K}=\mathscr{H}$. Furthermore $\mathbf{C 1}_{\mathscr{X}} \otimes \mathscr{L}(\mathscr{M})=\left(\mathfrak{U}^{\prime \prime} E\right)^{\prime}=\mathfrak{U}^{\prime} E ;$ so $\left.P\right|_{c_{X} \otimes \mathscr{L}(\mathscr{M})}$ is the normalized trace $\tau$. Thus $P_{E}=\imath \otimes \tau$ by Proposition 6.2 of Størmer (1979) where $\iota$ denotes the identity map on $\mathscr{L}(\mathscr{K})$. It follows as in the previous paragraph that either $\operatorname{dim} \mathscr{K}=1$ or $\operatorname{dim} \mathscr{M}=1$. If $\operatorname{dim} \mathscr{K}=1$ then $\mathfrak{A}^{\prime \prime} E=\mathbf{C} E$ and since $\mathfrak{A}^{\prime \prime} E \simeq \mathfrak{A}^{\prime \prime} F$ one has $\mathfrak{A}^{\prime \prime} F=\mathbf{C} F$. But $\mathfrak{A}$ is a JW-factor and hence it is not possible that

$$
\mathfrak{A}^{\prime \prime}=\mathbf{C} E \oplus \mathbf{C} F \text {. }
$$

Thus $\operatorname{dim} \mathscr{K} \neq 1$ and one must have $\operatorname{dim} \mathscr{M}=1$. In this latter case

$$
E \mathscr{L}(\mathscr{H}) E=\mathfrak{A}^{\prime \prime} E=[\mathfrak{U}] E
$$

and by symmetry $F \mathscr{L}(\mathscr{H}) F=\mathfrak{A}^{\prime \prime} F=[\mathfrak{A}] F$. Since $\mathfrak{A}^{\prime \prime} E \simeq \mathfrak{A}^{\prime \prime} F, \operatorname{dim} E=\operatorname{dim} F$ and a simple modification of the proof of Lemma 6.3 in Størmer (1979) establishes that

$$
P(E A F+F A E)=0
$$

for all $A \in \mathscr{L}(\mathscr{H})$. But then $E A F \in \mathfrak{g}$ and the same argument used to prove that $P$ was faithful allows one to conclude this case is also impossible. Therefore $\mathfrak{U}^{\prime \prime}$ 
cannot have exactly two central projections different from 1 and the proof is complete.

COROLlaRy 5. Let $\mathscr{H}$ be an infinite-dimensional complex Hilbert space and $P$ a positive normal identity preserving projection of $\mathscr{L}(\mathscr{H})$ into itself such that $\mathfrak{U}=P\left(\mathscr{L}(\mathscr{H})_{h}\right)$ is a JC-algebra and $P \neq \imath$. Let $\mathrm{g}$ denote the null space of $P$.

The following conditions are equivalent

1. $\mathfrak{g}$ is a Lie algebra

2. $\mathfrak{g}$ is a Lie algebra satisfying

$$
\mathfrak{g} \circ \mathfrak{g} \subseteq P(\mathscr{L}(\mathscr{H}))
$$

3. There exists a *-anti-automorphism $\alpha$ of order 2 of $\mathscr{L}(\mathscr{H})$ such that

$$
P=(\iota+\alpha) / 2 \text {. }
$$

Proof. Clearly $3 \Rightarrow 2 \Rightarrow 1$. But condition 1 and Theorem 4 imply that $\mathfrak{g}=B_{\infty}$ or $C_{\infty}$ and $\mathfrak{A}$ is irreducible. Thus $P$ is the unique normal projection of $\mathscr{L}(\mathscr{H})$ onto [थ] , by Lemma 6.1 of Størmer (1979), and hence $P=(\iota+\alpha) / 2$ and $\alpha$ the *antiautomorphism of order 2 of $\mathscr{L}(\mathscr{H})$ such that $\mathfrak{A}=\left\{A ; A \in \mathscr{L}(\mathscr{H})_{h}, \alpha(A)=A\right\}$.

Remark. Adopt the assumptions of the above corollary. For $\mathfrak{g}$ to be a Lie algebra it is necessary that $\mathfrak{A}$ is irreducible and $\iota-P$ is a contraction, for example $\|\imath-P\|=\|(\imath-\alpha) / 2\| \leqslant 1$ and it appears that these conditions are sufficient. For example, if $P$ is a projection from $\mathscr{L} \mathbf{C}(\mathscr{H})$ into $\mathscr{L}(\mathscr{H})$ and if one assumes that $P(\mathscr{L C}(\mathscr{H})) \subseteq \mathscr{L} \mathrm{C}(\mathscr{H})$ then Proposition 7.7 of Arazy and Friedman (1978), and the assumption $P(1)=1$, imply that either

$$
(P(A)=E A E+(1-E) A(1-E)
$$

for some projection $E \in \mathscr{L}(\mathscr{H})$ or $P=(\imath+\alpha) / 2$ for some *-anti-automorphism of order 2. But in the first case $\mathfrak{A}$ is reducible unless $E=1$ or 0 and $P=\imath$. Thus only the possibility $P=(\iota+\alpha) / 2$ remains.

\section{References}

J. Arazy and Y. Friedman (1978), 'Contractive projections in $C_{1}$ and $C_{\infty}$ ', Mem. Amer. Math. Soc. 200.

E. Effros and E. Størmer (1979) 'Positive projections and Jordan structure in operator algebras', Math. Scand., to appear.

P. Jordan, J. von Neumann and E. Wigner (1934), 'On an algebraic generalization of the quantum mechanical formalism', Ann. of Math. 35, 29-64.

E. Størmer (1966), 'Jordan algebras of type 1', Acta Math. 115, 165-184. 
E. Størmer (1968), 'Irreducible Jordan algebras of self-adjoint operators', Trans. Amer. Math. Soc. 130, 153-166.

E. Størmer (1972), 'On projection maps of von Neumann algebras', Math. Scand. 30, 46-50.

E. Størmer (1979), 'Decomposition of positive projections on $C^{*}$-algebras', preprint.

J. Tomiyama (1959), 'On the projection of norm one in $W^{*}$-algebras. III', Tôhoku Math. $J$. 11, 125-129.

Z-X Wan (1975), Lie algebras (Pergamon Press, Oxford).

Department of Pure Mathematics

University of New South Wales

PO Box 1, Kensington 2033

Australia
Institute of Mathematics

University of Oslo

PO Box 1053, Blindern, Oslo 3

Norway 